\title{
Continuously tunable diamond Raman laser for resonance laser ionization
}

\author{
Katerina Chrysalidis, ${ }^{1,2, *}$ (i) Valentin N. Fedosseev, ${ }^{1}$ Bruce A. Marsh, ${ }^{1}$ Richard P. Mildren, ${ }^{3}$ (i) \\ David J. Spence, ${ }^{3}$ (1) Klaus D. A. Wendt, ${ }^{2}$ Shane G. Wilkins, ${ }^{1}$ and Eduardo Granados ${ }^{1}$ \\ ${ }^{1}$ CERN, 1217 Geneva, Switzerland \\ ${ }^{2}$ Institut für Physik, Johannes Gutenberg-Universität, 55099 Mainz, Germany \\ ${ }^{3}$ MQ Photonics Research Centre, Macquarie University, NSW 2109, Australia \\ *Corresponding author: katerina.chrysalidis@cern.ch
}

Received 7 May 2019; accepted 26 June 2019; posted 10 July 2019 (Doc. ID 366526); published 6 August 2019

We demonstrate a highly efficient, tunable, $\sim 5 \mathrm{GHz}$ linewidth diamond Raman laser operating at $479 \mathrm{~nm}$. The diamond laser was pumped by a wavelength-tunable intracavity frequency-doubled titanium sapphire (Ti:Sapphire) laser operating at around $450 \mathrm{~nm}$, at a repetition rate of $10 \mathrm{kHz}$ with a pulse duration of $50 \mathrm{~ns}$. The Raman resonator produced a continuously tunable output with high stability, high conversion efficiency $(28 \%)$, and beam quality $\left(\mathrm{M}^{2}<1.2\right)$. We also demonstrate that the linewidth and tunability of the pump laser is directly transferred to the Stokes output. Our results show that diamond Raman lasers offer great potential for spectroscopic applications, such as resonance laser ionization, in an all-solid-state platform. (c) 2019 Optical Society of America

https://doi.org/10.1364/OL.44.003924

Provided under the terms of the OSA Open Access Publishing Agreement

At the CERN-ISOLDE Radioactive Ion Beam (RIB) Facility, a large variety of (radioactive) isotopes are produced with the isotope separator on-line (ISOL) technique in order to study their fundamental nuclear and atomic properties [1]. The ISOLDE Resonance Ionization Laser Ion Source (RILIS) is the most selective of the ion sources. It is based on stepwise resonance excitation and ionization of atoms, making use of elementspecific energy level configurations [2]. For efficient ionization, the linewidths are matched to the Doppler-broadening of the atomic lines (including fine and hyperfine structure splittings), usually lying in the range of $5-15 \mathrm{GHz}$ (see [2]). Additionally, in order to account for the various atomic configurations, a broad spectral coverage across the UV to IR range is required. Laser systems operating at a repetition rate of $10 \mathrm{kHz}$ are used to ensure that each atom is subject to ionization before exiting the hot cavity [3]. The required laser power depends on the chosen atomic transition and can range from $\mathrm{mW}$ to $\mathrm{W}$.

Currently, the strategy used at the ISOLDE RILIS that meets all the aforementioned specifications comprises the use of a dual-laser system with nanosecond titanium sapphire (Ti:Sapphire) and dye lasers. The spectral range is increased by frequency-mixing stages capable of generating virtually any wavelength between 210-950 nm (see [2,4]). Even though this solution meets all the specifications, it can require high operational costs. Especially, UV-pumped dyes require frequent changes (up to once every $10 \mathrm{~h}$ ) for maintaining adequate power output. This is detrimental for the operation of the experimental facility over many days or weeks. Therefore, a strong interest exists in finding a simple and compact all-solid-state laser alternative that can provide a widely tunable output across the spectrum at the required repetition rate.

A potential solution relies on the use of stimulated Raman scattering (SRS) in combination with tunable Ti:Sapphire lasers to access the spectrum traditionally occupied by UV-pumped dye lasers (in particular, the $475-540 \mathrm{~nm}$ band). During the last five decades, SRS, resulting from a photon-phonon inelastic interaction in Raman-active media [5], has established itself as a versatile method to generate wavelengths that are otherwise difficult to produce. When compared to optical parametric oscillators (OPOs) and other nonlinear (often tedious and inefficient) conversion techniques like frequency mixing (see e.g., [6]), Raman lasers are simple to construct, do not require management of phase matching, and can be operated at any chosen repetition rate [7].

A large number of Raman-active materials have been investigated to date, showing stark differences in terms of wavelength shift, transparency, phonon-dephasing time, or Raman gain, to name a few $[8,9]$. Traditionally, the attention has been focused on the enhancement of the conversion efficiency and wavelength coverage. Three main factors led to the choice of diamond in the work presented here: the large wavelength shift of $1332.5 \mathrm{~cm}^{-1}$, which converts the $450 \mathrm{~nm}$ pump laser directly to $479 \mathrm{~nm}$ in a single Stokes shift; its high Raman gain at visible wavelengths $(50 \mathrm{~cm} / \mathrm{GW}$ at $532 \mathrm{~nm}$ [10]); and ultrabroad transparency from the mid-IR [11] down to deep UV wavelengths [12]. With the advent of the continuous-wave (CW) narrow-linewidth single-longitudinal-mode (SLM) diamond Raman laser, detailed experimental studies regarding the emission linewidth of Raman converters have been published [13-15]. However, studies regarding the linewidth characteristics of highly efficient nanosecond broadband diamond Raman lasers, which are suitable candidates for resonance laser ionization experiments, remain scarce in the literature. 
The aim of the present study is to demonstrate that the output of broadly tunable solid-state lasers can be efficiently Raman shifted to new wavelengths maintaining the pump laser linewidth. In our experiments, we employed a widely tunable, frequency-doubled Ti:Sapphire laser at $450 \mathrm{~nm}$ for generating the first Stokes order inside a diamond at around $479 \mathrm{~nm}$. We demonstrate a continuously tunable output with a conversion efficiency of $>28 \%$ while maintaining a constant linewidth of $\sim 5 \mathrm{GHz}$ in the 475-500 $\mathrm{nm}$ spectral range.

Figure 1 shows a schematic diagram of the experimental setup. The pump light at $450 \mathrm{~nm}$ was provided by an intracavity frequency-doubled Ti:Sapphire laser (Z-cavity layout; for more details see [16]). It was pumped by the second harmonic of a Q-switched Nd:YAG laser (Innolas Nanio 532-20$\mathrm{V})$ producing up to $18 \mathrm{~W}$ of $532 \mathrm{~nm}$ light at $10 \mathrm{kHz}$ repetition rate, of which $8 \mathrm{~W}$ were used. The resulting pulse length of the Ti:Sapphire is $50 \mathrm{~ns}$ long, with a smooth temporal profile of Gaussian shape. The second harmonic of the Ti:Sapphire laser was efficiently produced by intracavity frequency-doubling, and exhibited a TEM ${ }_{00}$ Gaussian mode with an $\mathrm{M}^{2}<1.3$. The efficiency of second-harmonic generation was optimized by placing the crystal in one of the arms of the Ti:Sapphire resonator with a relatively small beam diameter. Frequency selection and tunability is obtained by using a birefringent filter and a thin $(0.3 \mathrm{~mm})$ Fabry-Perot etalon while frequency conversion is achieved within a $6 \mathrm{~mm}$ thick bismuth borate $\left(\mathrm{BiB}_{3} \mathrm{O}_{6}\right)$ crystal.

With this configuration, the system was capable of producing approximately $1.2 \mathrm{~W}$ of average power at $450 \mathrm{~nm}$. This output was focused into the diamond crystal by a $100 \mathrm{~mm}$ focal length lens, producing a waist of $57 \pm 5 \mu \mathrm{m}$ in diameter. The linewidth (FWHM) of the $450 \mathrm{~nm}$ light was measured with a high-precision laser radiation spectrum analyzer and wavelength meter LM-007 (CLUSTER LTD Moscow) as $6.5 \pm 0.9 \mathrm{GHz}$. This linewidth is about a factor of $\sqrt{2}$ wider than the fundamental linewidth of the Ti:Sapphire laser.

A synthetic diamond crystal (low-birefringence, low-nitrogen, chemical vapor deposition (CVD)-grown single crystal, Element Six Ltd.) with dimensions of $8 \mathrm{~mm}$ (length) $\times 2 \mathrm{~mm}$ (width) $\times$ $2 \mathrm{~mm}$ (height), plane-cut for beam propagation along the $\langle 110\rangle$ axis, was selected as the Raman laser medium. In order to access the highest Raman gain coefficient, the fundamental and Stokes beams were copolarized along the $\langle 111\rangle$ axis [17] by means of a $\lambda / 2$-plate placed before the cavity (see Fig. 1). In order to avoid any potential etalon effects introduced by the uncoated Raman medium [18], the diamond was placed at an angle of $10^{\circ}$ with respect to the resonator mode. Incidence at Brewster's angle was

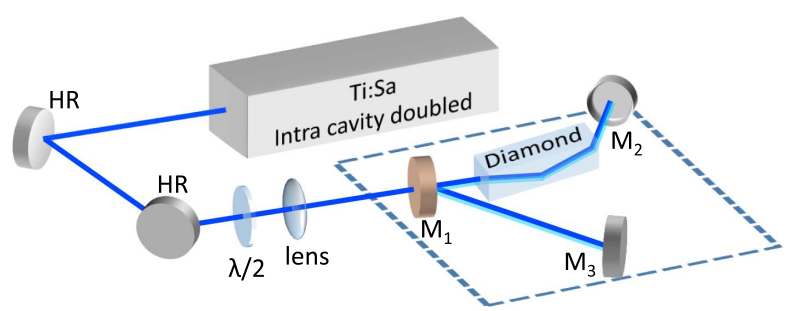

Fig. 1. Schematic layout of the Raman laser setup. An intracavity frequency-doubled Ti:Sapphire laser at $450 \mathrm{~nm}$ was used for pumping a diamond crystal. The cavity (dotted box) consists of three curved mirrors. The surface reflections of the diamond itself were used for coupling out the Raman converted laser light. also tested; however, this arrangement produced an ellipticity that significantly decreased the available pump intensity, therefore increasing the lasing threshold.

The diamond laser resonator was designed specifically for the pump pulse energy available $(\sim 65 \mu \mathrm{J})$. It is a stable cavity composed of three curved mirrors $\left(M_{1}, M_{2}\right.$, and $\left.M_{3}\right)$. The reported output power is the sum of the leakages through the mirrors and all four reflections off of the diamond crystal, thereby representing the single-beam power that could be obtained using low-transmission mirrors, antireflection coating on the diamond surfaces, and a single output coupler. The total output coupling was estimated to be approximately $63 \%$. Mirror $M_{1}$ is a dichroic curved mirror with a radius of curvature (ROC) of $500 \mathrm{~mm}$, a transmission of $60 \%$ at $450 \mathrm{~nm}$, and $>90 \%$ reflectivity at $479 \mathrm{~nm}$. Mirrors $\mathrm{M}_{2}$ and $\mathrm{M}_{3}$ are broadband high-reflecting mirrors (>99\%) with ROCs of $24 \mathrm{~mm}$ and $150 \mathrm{~mm}$, respectively.

The external resonator employed in the present study differs substantially from the previously reported extracavity nanosecond diamond Raman lasers $[17,19,20]$, where the pulse energy allowed for the use of larger resonator waist sizes for efficient laser operation. In our particular case, the pulse energy is comparatively low, while the pump pulse duration is several times longer. These facts enable the use of a stable cavity with a small waist and compact overall size.

The distances between the three curved mirrors was systematically adjusted in order to maximize the Stokes output power, obtaining a well-matched cavity waist of $\sim 60 \mu \mathrm{m}$ in diameter $\left(1 / e^{2}\right)$. The optimal distance between $M_{1}$ and $M_{2}$ was found to be approximately $48 \mathrm{~mm}$ (not including the refractive index of the diamond crystal), whereas the distance between $M_{1}$ and $M_{3}$ was set to $125 \mathrm{~mm}$. The total round trip time of the cavity was estimated to be $1.28 \mathrm{~ns}$, which is substantially less than the pump pulse duration of 50 ns, enabling efficient Raman conversion. Consequently, the measured slope efficiency for the first Stokes was $79 \%$, reaching a maximum conversion efficiency of 28\%, as shown in Fig. 2. The lasing threshold was $40 \mu \mathrm{J}$, and the maximum output power achieved $(183 \mathrm{~mW})$

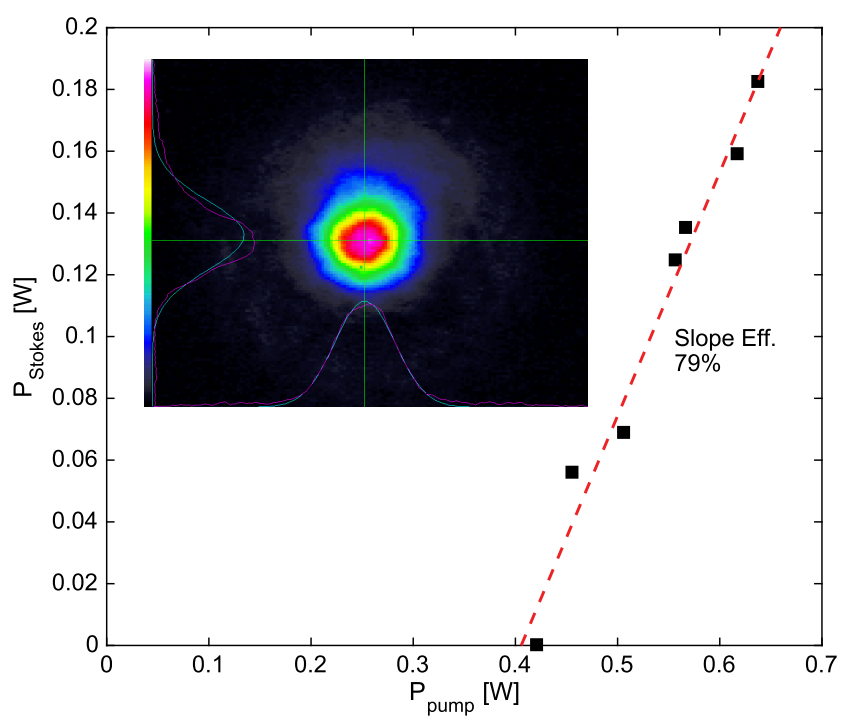

Fig. 2. Slope efficiency of the Raman laser presented here is $79 \%$ for the first Stokes order. The inset shows the near-field image of the first Stokes output. 
was limited only by the pump laser power. The output beam quality was measured using a beam profiler (Coherent Inc. LaserCam-HR II; see the inset of Fig. 2) to be $\mathrm{M}^{2}<1.2$, marginally smaller than the one measured for the Ti:Sapphire pump laser.

The coupling between pump and Stokes by SRS is a complex process, depending strongly on the spatial, spectral, and temporal arrangement of the fields involved. In general, the bandwidth of the laser fields relates to the time scale of amplitude and phase fluctuations. In our particular case, where the time scale of the fluctuations is much slower than the dephasing time $\left(T_{2}=5.7 \mathrm{a} € 7\right.$ ps for diamond [21]), the material excitation is considered to be in "steady state" [22]. This is also obvious when comparing the pump laser linewidth $\left(\Delta \omega_{P}=\right.$ $6.5 \mathrm{GHz})$ to the diamond spontaneous Raman scattering linewidth $\left(\Delta \omega_{R} \approx 45-57 \mathrm{GHz}[21]\right)$.

While the spontaneous Stokes noise fields may have a larger bandwidth than the pump laser, the Stokes output linewidth is driven to match the pump laser linewidth in the high-gain limit [22]. In this case, all the power in the emission linewidth of the pump laser is effective for amplifying the Stokes field. For resonance ionization applications, it is therefore preferred to operate with a relatively small spot (large gain and high output coupling) in order to converge rapidly to a linewidth similar to that of the pump laser.

The spectral characteristics of the Raman laser emission were studied using a LM-007 and a HighFinesselÅngstrom WS/6 wavelength meter with an absolute accuracy of $100 \mathrm{MHz}$ and $600 \mathrm{MHz}$, respectively. The principal component of the LM-007 wavelength meter is a block of four thermostabilized neon-filled Fizeau interferometers that have different bases $(3 \mu \mathrm{m}, 50 \mu \mathrm{m}, 1.5 \mathrm{~mm}$, and $40 \mathrm{~mm})$. In this work, the laser line profiles, a convolution of the first Stokes linewidth and instrumental width was measured using interferometer 3, which has an instrumental width of $\sim 5.3 \mathrm{GHz}$ and a resolution of $<1 \mathrm{GHz}$. Multiple output spectra were measured and averaged for different pump power conditions between $0.4-0.65 \mathrm{~W}$ (corresponding to $56-183 \mathrm{~mW}$ output power), and the results are shown in Fig. 3. As can be seen, the output had a smooth Lorentzian line shape and a linewidth of around $5 \pm 1 \mathrm{GHz}$, independent of the pump power used.

The results are consistent with the expected output linewidth when approaching the high Raman gain limit regime. The output Stokes linewidth is slightly narrower than the pump laser linewidth for all measured powers, and a factor of 10 times smaller than the spontaneous Stokes noise field. In addition, the measured Stokes linewidth is down to $4 \mathrm{GHz}$ for low pump power conditions and slightly increasing up to $5.8 \mathrm{GHz}$ at maximum pump power. For the case of relatively narrow pump linewidth $\left(\Delta \omega_{P} \ll \Delta \omega_{R}\right)$, the initially broad Stokes noise spectrum with $\Delta \omega_{S} \approx 57.5 \pm 6 \mathrm{GHz}$ is gain-narrowed by the factor $\left[\ln 2 /\left(g_{0} I_{P} z\right)\right]^{1 / 2}$, where $g_{0}$ is the gain coefficient, $I_{P}$ is the averaged pump intensity, and $z$ is propagation distance [23]. Assuming $g_{0}=50 \mathrm{~cm} / \mathrm{GW}$, an average pump intensity of $I_{P}=25 \mathrm{MW} / \mathrm{cm}^{2}$, the amplified Stokes noise linewidth after a few passes through the diamond is already smaller than the pump laser linewidth (the cavity photon lifetime is $\sim 1.2 \mathrm{~ns}$ at $479 \mathrm{~nm}$ ). The gain at threshold, however, is already high enough to make the Stokes spectrum closely duplicate the one of the pump, with a degree of similarity expected to get closer as the gain increases towards full output power.

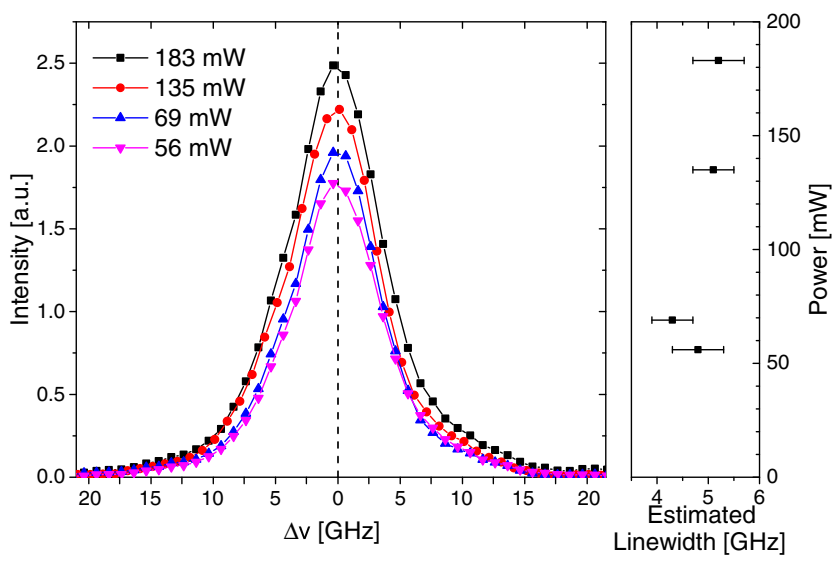

Fig. 3. On the left, the central peaks of the LM-007 wavelength meter, Fizeau interferometer \#3 (base $1.5 \mathrm{~mm}$ ), recorded at different output powers are shown. The intensity has been scaled relative to the pump power. On the right, the results of the linewidth measurements from fitting the spectra taken with the LM-007 are shown for the different pump powers. More details can be found in the text.

The output wavelength was continuously tuned by adjusting the pump laser center wavelength using a thin etalon. Even though the tunable range of the pump laser covers the 350$475 \mathrm{~nm}$ range, the results shown represent only a small tuning range that covers $450.28-450.33 \mathrm{~nm}$ (corresponding to a shifted output between $479.02-479.08 \mathrm{~nm}$ ) in order to study in detail the output Stokes linewidth variation when tuning through the Stokes cavity free spectral range (FSR) and the full linewidth of the pump laser. Stokes spectra were measured continuously while the pump laser was tuned in power and center wavelength simultaneously.

Figure 4 shows the 1 st Stokes wavenumber and measured linewidth (at FWHM) for a range of pump wavelengths at different pump powers. These scans were performed in a random manner, without adjustments of the Raman cavity, in order to test the versatility and stability of the technique. Consequently, the data are scattered unevenly across the plot. Continuous output tunability was achieved due to the small frequency spacing of the fundamental cavity modes (FSR $\approx 815 \mathrm{MHz}$ ). This is apparent in Fig. 4 as a linewidth variation of $\sim 1 \mathrm{GHz}$ in the Stokes output during tuning.

It is important to note that the effect of the cavity FSR can become detrimental when shorter resonators are employed. In general, the resonator length is designed for efficient Raman conversion. For nanosecond pump pulses, the resonator is usually as short as possible in order to maximize the number of passes through the gain media. Since the Raman effect is free of spatial hole burning [24], for cavities with a FSR approaching the pump linewidth, the output Stokes is usually composed of an ensemble of well-spaced longitudinal modes, which is not effective for resonance ionization applications.

The results demonstrate that a synthetic low-birefringence diamond is suitable for realizing highly efficient, linewidth invariant, Raman shifters across the visible spectrum. The linewidth behavior is dictated by the specific properties of the pump source and the characteristics of the gain medium and resonator design. Here, we propose a Raman laser system designed for spectroscopic applications that require large 


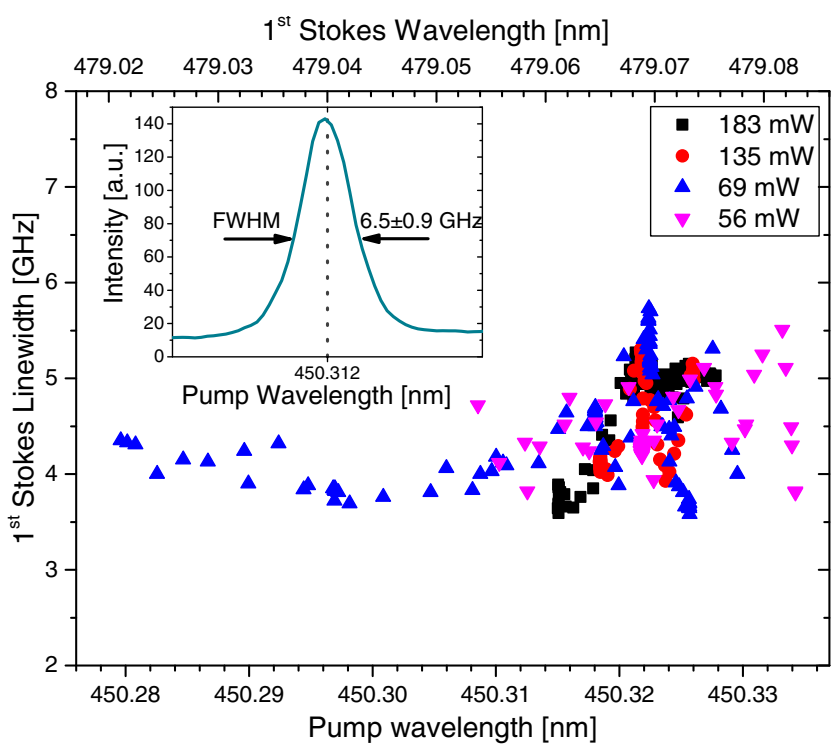

Fig. 4. Linewidth measurement of the 1st Stokes order using a HighFinesse/Angstrom WS/6, taken at different output powers, while adjusting the pump wavelength. The inset shows the pump linewidth measured with the LM-007 wavelength meter at a fixed wavelength.

tunability and a specific linewidth in the low $\mathrm{GHz}$ range. Furthermore, the presented work shows that the capabilities of solid-state laser systems, as used, e.g., at the ISOLDE RILIS, can be easily expanded by diamond Raman lasers. The spectral coverage can be significantly improved, without drawbacks concerning the reliability or performance of the installation. The reduction in operational requirements opens up new possibilities for using ionization schemes containing wavelengths between $475-540 \mathrm{~nm}$. This is of special importance for facilities that are not equipped with a dual Ti:Sapphire/dye laser systems (e.g., [25-27]).

Further optimizations of the Raman laser cavity design are foreseen, aiming for an easy-to-use, wavelength-independent add-on for the Ti:Sapphire lasers. Steps towards this have been taken, simplifying the current cavity layout significantly. It is expected that for the end of the CERN long shutdown 2 (LS2), such a system will be available for standard use at the RILIS laboratory.

Funding. Horizon 2020 Framework Programme (H2020) (654002).

\section{REFERENCES}

1. R. Catherall, W. Andreazza, M. Breitenfeldt, A. Dorsival, G. J. Focker, T. P. Gharsa, T. J. Giles, J. L. Grenard, F. Locci, P. Martins, S. Marzari, J. Schipper, A. Shornikov, and T. Stora, J. Phys. G 44, 094002 (2017).
2. V. Fedosseev, K. Chrysalidis, T. Day Goodacre, B. Marsh, S. Rothe, C. Seiffert, and K. Wendt, J. Phys. G 44, 084006 (2017).

3. V. Mishin, V. Fedosseev, H.-J. Kluge, V. Letokhov, H. Ravn, F. Scheerer, Y. Shirakabe, S. Sundell, and O. Tengblad, Nucl. Instrum. Methods Phys. Res. Sect. B 73, 550 (1993).

4. S. Rothe, T. Day Goodacre, D. Fedorov, V. Fedosseev, B. Marsh, P. Molkanov, R. Rossel, M. Seliverstov, M. Veinhard, and K. Wendt, Nucl. Instrum. Methods Phys. Res. Sect. B 376, 91 (2016).

5. R. Loudon, Adv. Phys. 13, 423 (1964).

6. P. Naubereit, J. Marín-Sáez, F. Schneider, A. Hakimi, M. Franzmann, T. Kron, S. Richter, and K. Wendt, Phys. Rev. A 93, 052518 (2016).

7. H. Pask, Prog. Quantum Electron. 27, 3 (2003).

8. T. T. Basiev, A. A. Sobol, P. G. Zverev, V. V. Osiko, and R. C. Powell, Appl. Opt. 38, 594 (1999).

9. J. A. Piper and H. M. Pask, IEEE J. Sel. Top. Quantum Electron. 13, 692 (2007).

10. D. J. Spence, E. Granados, and R. P. Mildren, Opt. Lett. 35, 556 (2010).

11. A. Sabella, J. A. Piper, and R. P. Mildren, Opt. Lett. 39, 4037 (2014).

12. E. Granados, D. J. Spence, and R. P. Mildren, Opt. Express 19, 10857 (2011).

13. O. Lux, S. Sarang, O. Kitzler, D. J. Spence, and R. P. Mildren, Optica 3, 876 (2016).

14. X. Yang, O. Kitzler, D. J. Spence, R. J. Williams, Z. Bai, S. Sarang, L. Zhang, Y. Feng, and R. P. Mildren, Opt. Lett. 44, 839 (2019).

15. D. J. Spence, O. Kitzler, J. Lin, H. M. Pask, S. C. Webster, N. Hempler, G. P. A. Malcolm, and R. P. Mildren, in European Conference on Lasers and Electro-Optics and European Quantum Electronics Conference (CLEO/Europe-EQEC) (Optical Society of America, 2017), paper CA_11_2.

16. S. Rothe, B. Marsh, C. Mattolat, V. Fedosseev, and K. Wendt, J. Phys. Conf. Ser. 312, 052020 (2011).

17. A. Sabella, J. A. Piper, and R. P. Mildren, Opt. Lett. 35, 3874 (2010).

18. G. Hernandez, Cambridge Studies in Modern Optics 3: Fabry-Perot Interferometers (Cambridge University, 1986).

19. R. P. Mildren, J. E. Butler, and J. R. Rabeau, Opt. Express 16, 18950 (2008).

20. R. P. Mildren and A. Sabella, Opt. Lett. 34, 2811 (2009).

21. K. Lee, B. J. Sussman, J. Nunn, V. Lorenz, K. Reim, D. Jaksch, I. Walmsley, P. Spizzirri, and S. Prawer, Diaond. Relat. Mater. 19, 1289 (2010).

22. D. J. Spence, Prog. Quantum Electron. 51, 1 (2017).

23. M. G. Raymer, J. Mostowski, and J. L. Carlsten, Phys. Rev. A 19, 2304 (1979).

24. O. Lux, S. Sarang, R. J. Williams, A. McKay, and R. P. Mildren, Opt. Express 24, 27812 (2016).

25. J. Lassen, P. Bricault, M. Dombsky, F. Izdebski, J. P. Lavoie, M. Gillner, T. Gottwald, F. Hellbusch, A. Teigelhoefer, A. Voss, K. D. A. Wendt, F. D. McDaniel, and B. L. Doyle, AIP Conf. Proc. 1099, 769 (2009).

26. N. Lecesne, R. Alvès-Condé, , E. Coterreau, F. De Oliveira, M. Dubois, J. L. Flambard, H. Franberg, T. Gottwald, P. Jardin, J. Lassen, F. Le Blanc, R. Leroy, C. Mattolat, A. Olivier, J. Y. Pacquet, A. Pichard, S. Rothe, M. G. Saint-Laurent, and K. Wendt, Rev. Sci. Instrum. 81, 02A910 (2010).

27. T. Sonoda, M. Wada, H. Tomita, C. Sakamoto, T. Takatsuka, T. Noto, H. limura, Y. Matsuo, T. Kubo, T. Shinozuka, T. Wakui, H. Mita, S. Naimi, T. Furukawa, Y. Itou, P. Schury, H. Miyatake, S. Jeong, H. Ishiyama, Y. Watanabe, and Y. Hirayama, Hyperfine Interact. 216, 103 (2013). 